\title{
Magnetic material optimization for hybrid vehicle PMSM drives
}

\author{
Sigrid Jacobs ${ }^{1}$, Dietrich Hectors ${ }^{3}$, François Henrotte ${ }^{2}$, Martin Hafner ${ }^{2}$ \\ Mercedes Herranz Gracia ${ }^{2}$, Kay Hameyer ${ }^{2}$, Patrick Goes ${ }^{3}$ \\ ${ }^{1}$ S. Jacobs (corresponding author), ArcelorMittal, Guldensporenpark 78, B-9820 Merelbeke, Belgium \\ ${ }^{2}$ IEM RWTH-Aachen University, Schinkelstr. 4, D-52056 Aachen, Germany \\ ${ }^{3}$ ArcelorMittal R\&D Gent, J. Kennedylaan 3, B-9060 Zelzate, Belgium
}

\begin{abstract}
The motivation of this analysis is the need of high efficiency and high power density permanent magnet synchronous motor (PMSM) drives for use in electrical vehicle power trains. It is clear that the chosen electrical steel for the lamination stack plays an important role, but proper quantification is missing. The purpose of this paper is to formalize the problem of selecting the optimal steel grade for the construction of PMSM's. This question is important to steel producers, not only for helping customers selecting the most appropriate existing grade for their application, but also for defining the strategic orientation of the further R\&D of enhanced electrical steel grades. The notion of steel efficiency is defined and, after describing the FE implementation of iron loss models, a methodology for material optimisation is proposed.
\end{abstract}

Efficiency, finite element calculation, materials, low loss fully processed electrical steels, motor design, optimization, permanent magnet motor, PHEV (plug in hybrid electric vehicle), series HEV.

\section{Introduction}

The development of hybrid and electric vehicle power trains set severe constraints on the electrical machines in terms of their volume and weight, which translate into the use of high efficiency and power density PMSM drives. ArcelorMittal is a steel supplier of electrical steels, classically mostly for large generators, industrial motors, traction machines and transformers, and wanted to define in more detail which grades were exactly needed for optimal performance of the PMSM for electric traction. Our current product range includes a variety of fully processed electrical steels (FP ES) with low losses, high permeability, high thermal conductivity, high yield strength, but the optimal trade off and steel gauge had to be clarified. Unfortunately, not much scientific publications dealing with this question can be found in the literature. Electrical designers focus on the global efficiency of the machines and do not usually adopt the point of view of the steel producer, hence this study.

Fig. 1 shows typical loss and polarisation levels of our offer in 0.35 and $0.50 \mathrm{~mm}$ FP grades These products are compared to the EN10106 which prescribes $50 \mathrm{~Hz}$ data guarantees. It is clear that for the PMSM characterisation, this frequency does not describe the materials sufficiently, so we have measurement data up to $10 \mathrm{kHz}$ available.

Electrical machine design is the conjunction of various, and sometimes contradictory objectives, e.g. maximizing the machine's total efficiency, which is the ratio of the mechanical power to the total input power:

$$
\eta=\frac{P_{M e c h}}{P_{\text {Mech }}+P_{F e}+P_{\text {Joule }}},
$$

and maximizing the torque to weight ratio. From the point of view of a steel producer, on the other hand, maximizing steel efficiency $\eta_{F e}$, defined as the ratio of the mechanical power $P_{M e c h}$ to the sum of the mechanical power and the iron 


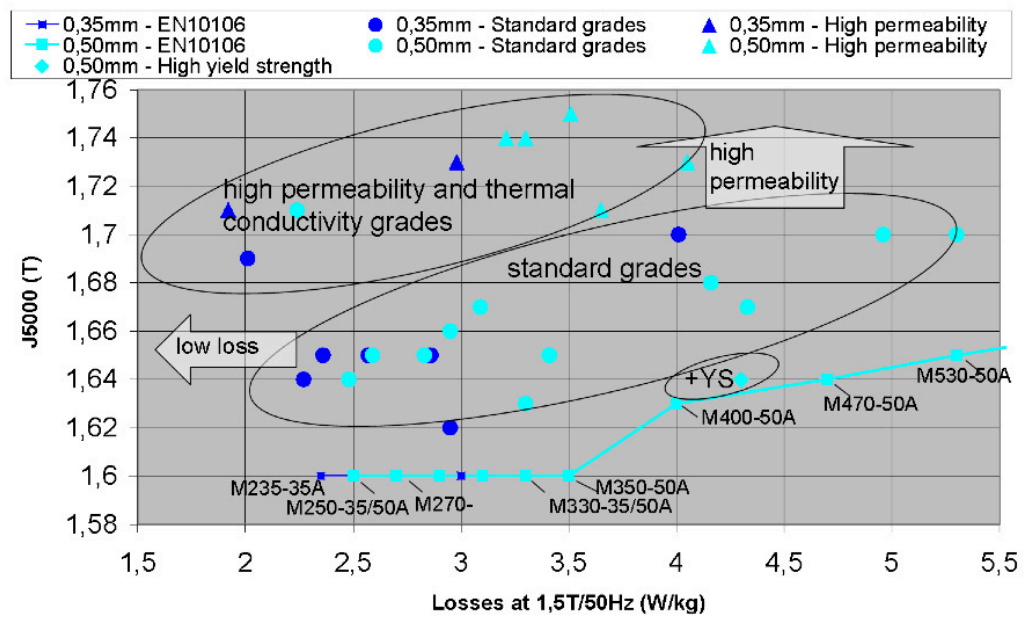

Figure 1: Losses versus polarisation of the ArcelorMittal offer in $0.50 \mathrm{~mm}$ (light symbols) and $0.35 \mathrm{~mm}$ (dark symbols) gauge, compared to the requirements of the EN10106.

losses $P_{F e}(2)$, seems to be a legitimate objective as well.

$$
\eta_{F e}=\frac{P_{M e c h}}{P_{M e c h}+P_{F e}}=\frac{1}{1+\frac{P_{F e}}{P_{M e c h}}}
$$

However, whereas iron losses increase with frequency and current, finite element simulations carried out on a PMSM machine show that the steel efficiency increases with the current, but decreases when the frequency increases, because the mechanical power increases slower than iron losses. The two objectives (maximizing total efficiency and maximizing steel efficiency, i.e. minimizing the ratio $P_{F e} / P_{M e c h}$ ) have been graphically represented by white arrows in Fig. 2. For a better visualization, the ratio $P_{F e} / P_{M e c h}$ has been plotted instead of $\eta_{F e}$; the smaller this ratio, the better the steel efficiency $\eta_{F e}(2)$. Fig. 2 shows that the steel efficiency reaches a maximum lower right corner of the diagram, i.e. in an operation range with low frequency and high currents where the overall efficiency of the machine is very bad. The two objectives are thus clearly contradictory; they have nearly opposite gradients in the operation space of the PMSM motor (which is the space supply current $I$ vs working frequency $f$ ), and the question arises which trade off will yield the optimum motor design.

Clearly the overall efficiency $\eta$ must remain the deciding factor, but still there is a potential for energy saving and efficiency improvement through an optimum choice of magnetic material. The purpose of this paper is to formalize this problem, which is important to steel producers, not only for helping customers selecting the most appropriate grade for their application but also for defining the specifications of the electrical steels which should be further developed.

All computations have been carried on on a 5 pole pairs $25 \mathrm{~kW} 3000 \mathrm{rpm}$ permanent magnet synchronous machine (PMSM) with buried magnets and concentrated windings, Fig. 3 . This is a typical machine for use in a hybrid electrical vehicle drive.

\section{Iron loss models}

For this purpose, it is required to evaluate iron losses in a PMSM motor on basis of different material characteristics. Measured material characteristics cannot in general be directly used in FE programs. A parametric iron loss model must first be determined, of which the free parameters are identified by correlation with the available measurements.

A widely accepted iron loss model is the model of Bertotti [1] :

$$
W_{\text {Ber }}=p_{\text {hyst }}+p_{\text {eddy }}+p_{\text {excess }},
$$

with the hysteresis losses

$$
p_{\text {hyst }}=k_{h}\left(1+\frac{B_{\min }}{B_{\max }}(r-1)\right) B_{\max }^{2} f
$$

the eddy curent losses

$$
p_{e d d y}=k_{e c} \sum_{n=1}^{\infty}\left|\vec{B}_{n}\right|^{2}(n f)^{2} \quad, \quad k_{e c}=\frac{\pi^{2} d^{2}}{6 \rho \rho_{e}}
$$

and the excess losses

$$
p_{\text {excess }}=k_{e x} \sum_{n=1}^{\infty}\left|\vec{B}_{n}\right|^{1.5}(n f)^{1.5}
$$




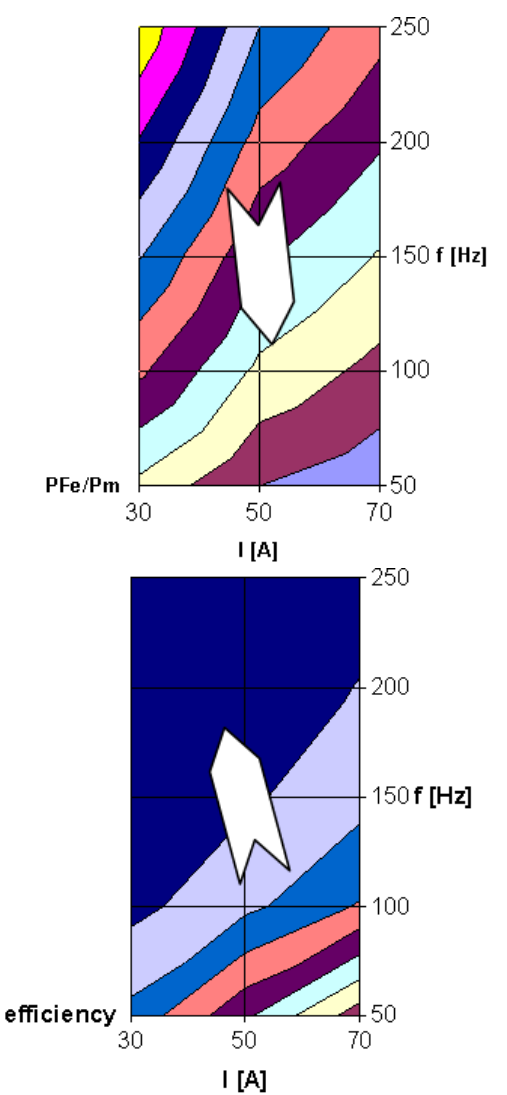

Figure 2: Ratio $P_{F e} / P_{M e c h}$ (above, range $[0.6 \%, 2.8 \%]$, each division is $0.2 \%$ ) and total efficiency of the machine $\eta$ (below, range [ $88 \%, 97 \%$ ], each division is $1 \%$ ), as a function of the supply current $I$ and the frequency $f$. The objectives of maximizing the motor's efficiency and the steel efficiency are contradictory, as indicated by the white arrows.

in terms of the Fourier decomposition of the periodic induction field (period $T, \omega=2 \pi / T$ ):

$$
\vec{B}(t)=\sum_{n=1}^{\infty} \vec{B}_{n} \cos \left(n \omega t+\varphi_{n}\right) .
$$

The multiplicative factor with parameter $r$ in (4) represents the effect of rotational hysteresis losses in the system. $B_{\min }$ and $B_{\max }$ are respectively the minimum and maximum values of $|\vec{B}(t)|$ over one period, Fig. 4 .

In experimental conditions (i.e. Epstein frame), the sinusoidal in time and unidimensional field $\vec{B}(t)$ is completely represented by the amplitude $\left|\vec{B}_{1}\right|$ of the fundamental harmonic, which is also the peak value $B_{\max }$ in this case. Bertotti's model writes thus

$$
W_{\text {Ber }}=k_{h} B^{2} f+k_{e c} B^{2} f^{2}+k_{e x} B^{1.5} f^{1.5}
$$

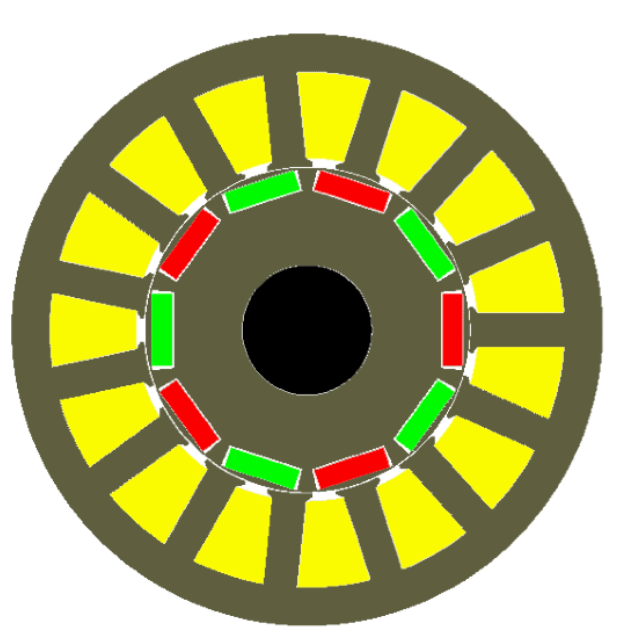

Figure 3: Cross section of the buried magnet PMSM machine.

with $B \equiv B_{\max }$.

This 2 parameter expression $\left(k_{h}\right.$ and $\left.k_{e c}\right)$ has been identified with measured iron loss characteristics $W_{\text {meas }}$ vs. $B \equiv B_{\max }$ with the following observations :

- The behaviour at large fields ( $B \leq 1$ Tesla) was poorly represented by (8) which contains at most a quadratic term in $B$, Fig. 5 .

- A higher order $B^{2+a_{3}}$ term must be introduced to obtain an accurate representation of iron losses at large fields. This term is probably caused by the magnetic saturation of the laminations [2], and will be called high order losses below.

- The exponent $a_{3}$ depends on the lamination thickness.

- The excess loss term in (8) turns out to be negligible $\left(k_{e x} \approx 0\right)$.

In consequence, the parameter identification has been done with the improved loss model

$$
W_{i m p}=a_{2} B^{2} f+\left(a_{1}+a_{4} B^{a_{3}}\right) B^{2} f^{2}
$$

with a very good accuracy over a large range of steel grades (N020, M235-35A, M250-50A, M250-50A, M330-50A, M330P-50A, M60050A, M310-65A, M800-100A, see Fig. 6) and a large range of frequencies around the nominal working frequency of the drive, $250 \mathrm{~Hz}$.

\section{Loss splitting}

After parameter identification, the models can be used to evaluate losses in the real situation, i.e. in the stator of a PMSM motor. The identified parameters can also be used in (4) and (5) with 


\begin{tabular}{l|lrrrrrrrr} 
& & $\mathrm{N} 020$ & $\mathrm{M} 235$ & $\mathrm{M} 250$ & $\mathrm{M} 330$ & $\mathrm{M} 330 \mathrm{P}$ & M600 & M310 & M800 \\
& & & $-35 \mathrm{~A}$ & $-50 \mathrm{~A}$ & $-50 \mathrm{~A}$ & $-50 \mathrm{~A}$ & $-50 \mathrm{~A}$ & $-65 \mathrm{~A}$ & $-100 \mathrm{~A}$ \\
\hline \hline a1 & & $3.50 \mathrm{E}-05$ & $6.00 \mathrm{E}-05$ & $1.14 \mathrm{E}-04$ & $1.30 \mathrm{E}-04$ & $1.35 \mathrm{E}-04$ & $1.60 \mathrm{E}-04$ & $1.30 \mathrm{E}-04$ & $2.50 \mathrm{E}-04$ \\
$\mathrm{a} 2$ & & $1.60 \mathrm{E}-02$ & $1.50 \mathrm{E}-02$ & $1.50 \mathrm{E}-02$ & $2.00 \mathrm{E}-02$ & $2.00 \mathrm{E}-02$ & $4.00 \mathrm{E}-02$ & $2.00 \mathrm{E}-02$ & $5.50 \mathrm{E}-02$ \\
a4 & & $5.00 \mathrm{E}-06$ & $5.50 \mathrm{E}-06$ & $1.70 \mathrm{E}-06$ & $1.70 \mathrm{E}-06$ & $8.00 \mathrm{E}-06$ & $1.20 \mathrm{E}-05$ & $3.00 \mathrm{E}-05$ & $2.10 \mathrm{E}-04$ \\
$\mathrm{a} 3$ & & 4 & 4 & 6 & 6 & 4 & 4 & 2 & 1 \\
\hline $\mathrm{d}$ & $m m$ & 0.2 & 0.35 & 0.5 & 0.5 & 0.5 & 0.5 & 0.65 & 1 \\
$\rho$ & $\mathrm{kg} / \mathrm{m}^{3}$ & 7630 & 7554 & 7554 & 7630 & 7769 & 7764 & 7554 & 7699 \\
$\rho_{E}$ & $\Omega m$ & $5.16 \mathrm{E}-07$ & $6.38 \mathrm{E}-07$ & $6.38 \mathrm{E}-07$ & $5.16 \mathrm{E}-07$ & $2.98 \mathrm{E}-07$ & $3.39 \mathrm{E}-07$ & $6.38 \mathrm{E}-07$ & $4.08 \mathrm{E}-07$ \\
\hline
\end{tabular}

Table 1: Identified coefficients for the parametric representation (9) of the iron loss characteristic of various steel grades.

\begin{tabular}{l|l|rrrrrrrr} 
& Unit & N020 & M235 & M250 & M330 & M330P & M600 & M310 & M800 \\
& & & $-35 A$ & $-50 A$ & $-50 A$ & $-50 A$ & $-50 A$ & $-65 A$ & $-100 A$ \\
\hline \hline Torque & Nm & 76 & 77 & 76 & 77 & 79 & 77 & 76 & 77 \\
\hline Basic & $\mathrm{W}$ & 159 & 193 & 272 & 357 & 438 & 504 & 324 & 761 \\
Harmonics & $\mathrm{W}$ & 8 & 13 & 25 & 26 & 29 & 23 & 29 & 57 \\
Rotational & $\mathrm{W}$ & 16 & 15 & 15 & 19 & 21 & 36 & 20 & 59 \\
High order & $\mathrm{W}$ & 73 & 81 & 83 & 101 & 164 & 213 & 126 & 568 \\
\hline Induced voltage & $\mathrm{V}$ & 258 & 260 & 256 & 262 & 271 & 264 & 256 & 260 \\
Input voltage & $\mathrm{V}$ & 263 & 265 & 261 & 267 & 276 & 269 & 261 & 266 \\
\hline Mechanical power & $\mathrm{W}$ & 24023 & 24275 & 24004 & 24221 & 24820 & 24337 & 23966 & 24125 \\
Joule losses & $\mathrm{W}$ & 564 & 564 & 564 & 564 & 564 & 564 & 564 & 564 \\
Iron losses & $\mathrm{W}$ & 255 & 302 & 395 & 505 & 652 & 777 & 500 & 1445 \\
Total losses & $\mathrm{W}$ & 819 & 866 & 959 & 1069 & 1216 & 1341 & 1064 & 2009 \\
\hline Steel efficiency & $\%$ & 98.9 & 98.8 & 98.4 & 98.0 & 97.4 & 96.9 & 98.0 & 94.3 \\
Efficiency & $\%$ & 96.7 & 96.6 & 96.2 & 95.8 & 95.3 & 94.8 & 95.8 & 92.3 \\
\hline
\end{tabular}

Table 2: Loss splitting performed for different steel grades at the nominal working point $(I=76 \mathrm{~A}$ and $f=250 \mathrm{~Hz})$ of the PMSM.

$k_{h}=a_{2}, k_{e c}=a_{1}$. One is thus left with parametric models for various loss components. Eddy current losses taking higher field harmonics into account or not, can be evaluated by means of (5), with or without truncating the sum in order to separate the losses associated with the fundamental harmonic from those associated with higher harmonics. Hysteresis losses are evaluated by (5) with $(r=2.5)$ or without $(r=1)$ rotational hysteresis. Finally, the losses associated with the additional high order term can be determined by (9) by setting the $a_{4}$ parameters to zero or not. The different terms of the loss splitting write then

- Basic: Basic hysteresis and eddy current losses

- Harmonics : Extra losses due to higher field harmonics

- Rotational: Extra losses due to rotational hysteresis

- High order: Extra losses represented by the high order term in (9).

Numerical values computed in nominal working conditions $(I=76 \mathrm{~A}, f=250 \mathrm{~Hz})$ are given in Table 2 for different steel grades.

\section{Material optimisation}

Our study has shown that the question of optimal material choice for a PMSM drive cannot be solved on basis of material considerations only. Steel efficiency is indeed a machine-related quantity. Moreover it cannot be optimized without consideration to the global efficiency of the motor. The optimum material is the one that offers minimum losses under typical operation of the machine. It is therefore a constrained optimisation problem, where the constraint is application dependent, which makes it necessary to incorporate information about the application in the analysis.

In the case of PMSM drives for hybrid vehicles, several stategies can be adopted according to the type of hybrid drive selected. We shall distinguish 3 situations, with increasing complexity. The simplest situation is when the machine is operated in a limited operation range around the nominal operation point, In this case, FE simulations in nominal situation are performed with different steel grades and the grade that yields the best efficiency $\eta$ is selected. In the case of Table 2, the optimum grades are the N020 and M235-35A, i.e. the thinnest and lowest loss 


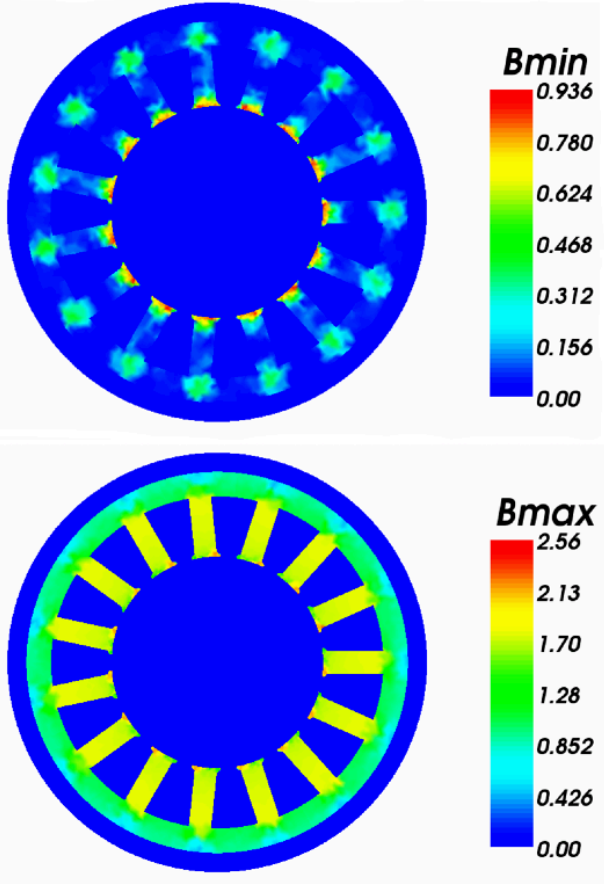

Figure 4: Distribution of $B_{\min }$ and $B_{\max }$ over the stator of the PMSM motor. The former indicate the general level of saturation, whereas the latter indicates locations where rotational hysteresis is important.

grades considered in this study. The results in Table. 2 show that both loss reduction and steel gauge reduction bring a contribution to the efficiencies as summarised in Tables 3 and 4.

The benefit of using high permeability FP ES is not straightforward, because the higher polarisation of the high perm grades results in higher flux levels for the same $H$ field, and a different exploitation point is reached. In the comparison shown in Table 5, this results in the fact that the higher perm version indeed realises a higher mechanical output, associated with the higher flux, but this is at the detriment of efficiencies, due to the fact that this new working point corresponds to higher a loss level. Hence, care needs to be taken when switching from standard to high perm grades. These material considerations conclusions are based on nominal calculations and these hold for the situations that prevail for the series hybrid concept.

For other hybrid vehicle types, however, the PMSM motor is operated over a larger area of the operation space. The efficiency map depicted Fig. 2 shows that the efficiency of the PMSM machine is optimum over a quite large region of the operation space (the dark blue region in Fig. 2). The machine must be controlled in order to be operated as much as possible inside this maximum efficiency region. With the knowledge of the motor only, and no further information about the way it is operated, the purpose of material op-
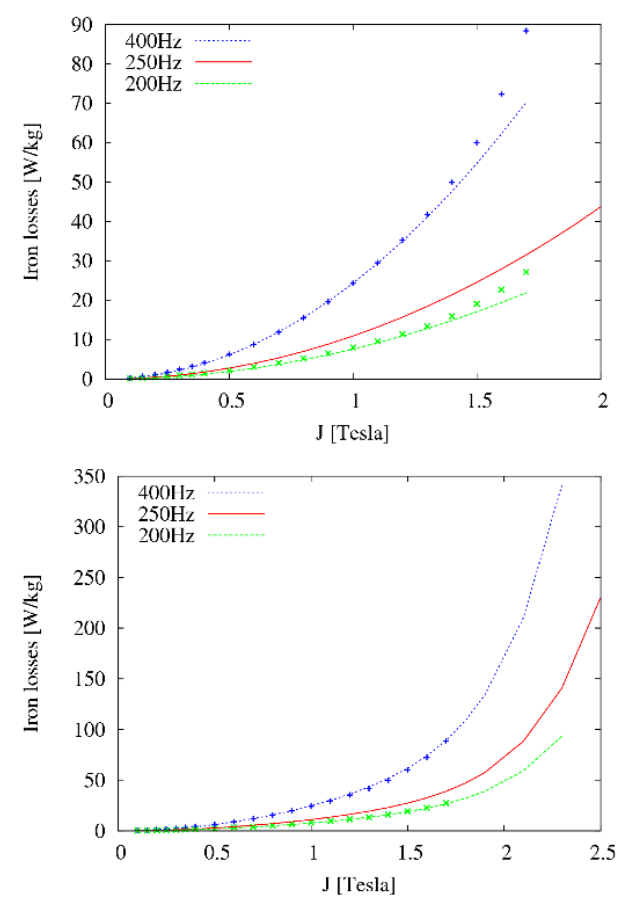

Figure 5: The lines represent iron losses according to Bertotti (8) (above) and with an additional higher order term (9) (below). The cross markers indicate measured values.

timisation is then to determine which grade provides the highest steel efficiency in average over the maximum efficiency region of the motor.

Finally the most sophisticated approach is to also take the way the machine is operated into consideration. One can rely for this on standard drive cycles, which can be translated into a statistical distribution $\operatorname{Prob}(I, f)$ over the operation space of the machine.

We have developped a method that allows combining efficiently the material information (loss and saturation characteristics) with a finite element model, and with the statistical distribution $\operatorname{Prob}(I, f)$ of the operation points of the motor, according to a given drive cycle, Fig. 8. This method involves an accurate computation of losses, including their breakdown into different components (Joule, eddy current, hysteresis) as decribed above, as well as an estimation of the influence of the presence of higher harmonics and of rotational hysteresis. The coupling of the finite element model with the statistical distribution $\operatorname{Prob}(I, f)$ is rendered tractable in terms of computation time by the extraction for each grade $\gamma$ under consideration, of a) the torque $T(I, \gamma)$, and b) histograms of peak induction in the machine, $\operatorname{Hist}\left(B_{\max }, I, \gamma\right)$ which give the volumic distribution of $B_{\max }$ over the finite elements of the PMSM model for a given steel grade $\gamma$, Fig. 7. For PMSM drives, these two charac- 


\begin{tabular}{l|rrr} 
& M250-50A & M330-50A & M600-50A \\
\hline \hline Steel efficiency & $98.4 \%$ & $98.0 \%$ & $96.9 \%$ \\
Global efficiency & $96.2 \%$ & $95.8 \%$ & $94.8 \%$ \\
\hline
\end{tabular}

Table 3: Impact on steel and global efficiency for nominal machine conditions when using $0.50 \mathrm{~mm}$ FP ES with different loss levels.

\begin{tabular}{l|rrrrr} 
& N020 & M235-35A & M250-50A & M310-65A & M600-50A \\
\hline \hline Steel efficiency & $98.9 \%$ & $98.8 \%$ & $98.4 \%$ & $98.0 \%$ & $94.3 \%$ \\
Global efficiency & $96.7 \%$ & $96.6 \%$ & $96.2 \%$ & $95.8 \%$ & $92.3 \%$ \\
\hline
\end{tabular}

Table 4: Impact on steel and global efficiency for nominal machine conditions when reducing the FP ES gauge at low loss levels.

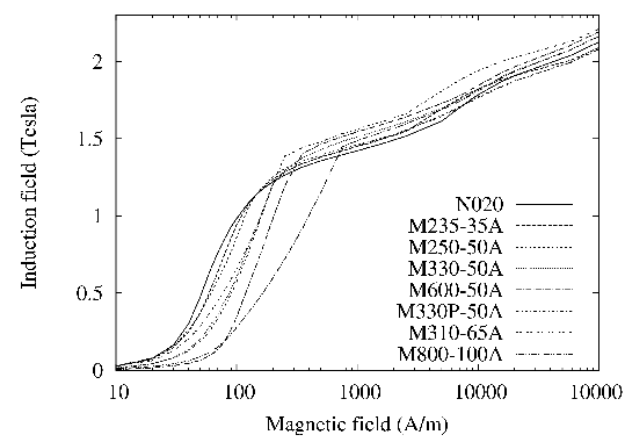

Figure 6: Saturation curves of the different steel grades considered.

teristics of the machine depend on the current $I$, and of course on the grade $\gamma$, but not on the frequency $f$. The distribution of iron losses over the operation space of the machine is then obtained by combination with the loss characteristic $W_{F e}(B, F, \gamma)$ of the steel grade under consideration

$$
\begin{aligned}
& P_{F e}(I, f, \gamma)= \\
& \int_{0}^{\infty} \rho \operatorname{Hist}(B, I, \gamma) W_{F e}(B, f, \gamma) d B
\end{aligned}
$$

and the iron losses under the chosen drive cycle are then given by integration over the operation space

$$
P_{F e}(\gamma)=\int P_{F e}(I, f, \gamma) \operatorname{Prob}(I, f) d I d f
$$

Similarly,

$$
P_{M e c h}(\gamma)=\int T(I, \gamma) 2 \pi f \operatorname{Prob}(I, f) d I d f .
$$

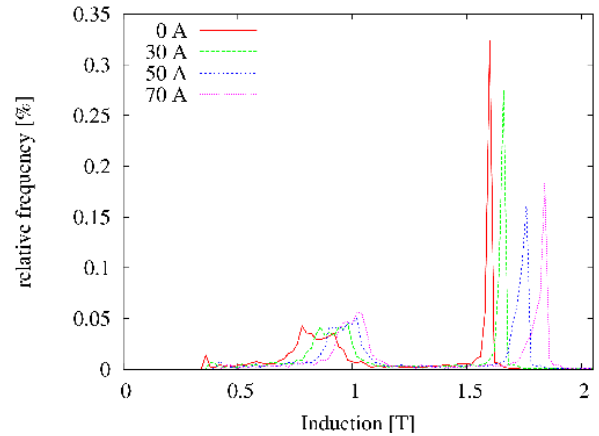

Figure 7: $\operatorname{Hist}(B, I, \gamma)$ : histogram of $B_{\max }$ in finite elements of the PMSM model, for a given material $\gamma$ and various values of the current $I$.

so that one can compare the steel efficiencies of different grades $\gamma, \eta_{F e}(\eta)$ in the drive conditions described by $\operatorname{Prob}(I, f)$.

At the end of the process, one is able to deliver quantitative statements about which material is the best adapted for a given drive system, in the sense that it gives the least iron losses $P_{F e}(\gamma)$ in average over the typical utilisation of the system. It is important to stress upon the fact that this optimum material is not necessarily the one with the lowest loss characteristic.

\section{Conclusion}

It is clear that, for the PMSM intended for hybrid traction, many developments are ongoing in order to achieve higher power density and higher efficiency machines. This study involved modelisation work to quantify the impact of the chosen FP ES in terms of the global efficiency of the PMSM, a well-known characteristic in electrical engineering, but introduced in parallel the steel efficiency. This steel efficiency is a useful param- 


\begin{tabular}{l|rrr} 
& M330-50A & M330P-50A & \\
\hline \hline Steel efficiency & $98.0 \%$ & $97.4 \%$ & \\
Global efficiency & $95.8 \%$ & $95.3 \%$ & $-0.5 \%$ \\
Mechanical power & $24221 \mathrm{~W}$ & $24820 \mathrm{~W}$ & $+2.5 \%$ \\
\hline
\end{tabular}

Table 5: Effect on efficiency and torque of the saturation level.

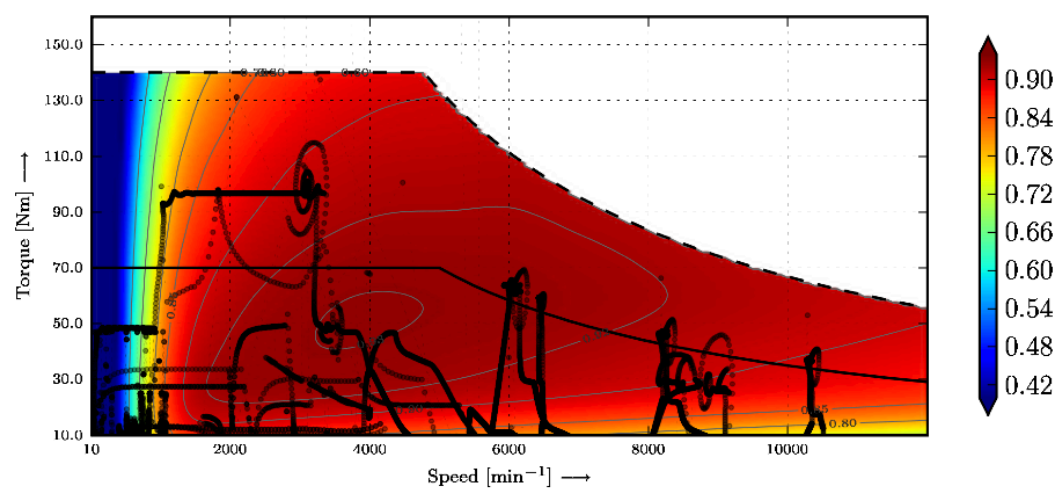

Figure 8: Efficiency map of a PMSM motor with superimposed sampled operation points of a typical drive cycle.

eter for electrical steel producers, for it allows assessing the impact on the machine performance of chosing one grade or another, and quantifying the consequence of this choice in terms of power.

Steel efficiency is a machine-related quantity. Finding the optimum ES requires therefore to incorporate information about the application, i.e. the drive, in the analysis. This is therefore a constrained optimisation problem. In the case of PMSM drives for hybrid vehicles, different stategies have been established to calculate the steel efficiency by means of FE simulations within reasonable computation time. Information about the type of hybrid vehicle and the drive cycles can be considered in the analysis.

In terms of ES impact, this study confirmed the need of Fully Processed grades with low high frequency loss. Low high frequency loss goes together with thin gauges for eddy current loss reduction. ArcelorMittal concluded that there was a need to give the PMSM constructor a more complete choice between the standardised NO20 and M235-35A grades. The development of $0.30 \mathrm{~mm}$ grades was chosen as a solution to further reduce the losses down from the $0.35 \mathrm{~mm}$ range, without going into the more difficult and higher cost machine construction methods associated with the use of $0.20 \mathrm{~mm}$ grades Our current offer of $0.30 \mathrm{~mm}$ grades consist of a M190P-30A, M200-30A and M230-30A. This offer presents a choice in loss, permeability, thermal conductivity and mechanical strength combinations, suited for the production of high performance PMSM for HEV's.

\section{References}

[1] G. Bertotti, General properties of power losses in soft ferromagnetic materials, IEEE Transactions on Magnetics, 24(1), pp. 621-630, January 1988.

[2] J. Gyselinck, L. Vandevelde, J. Melkebeek, P. Dular, F. Henrotte, W. Legros, Calculation of eddy currents and associated losses in electrical steel laminations, IEEE Transactions on Magnetics, 35(3), pp. 1191-1194, May 1999. 


\section{Authors}
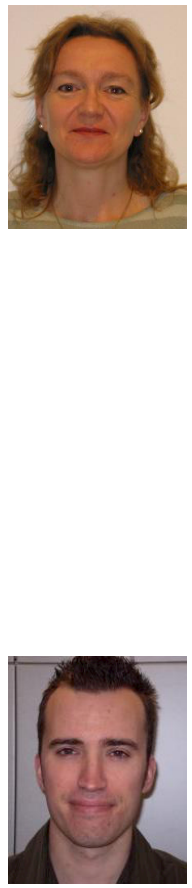

Sigrid Jacobs graduated in electrotechnical engineering at Ghent University in 1988 and obtained an MBA at the Vlerick School for management in 1991. After developing electrical steels at the metallurgy lab of the Ghent university, she joined the ArcelorMittal group and was involved in the coupling project of the coupled pickling-tandem mill line, the development of electrical steels at different plants from the group and is now a Product Development Engineer for the group's worldwide activities in Electrical Steels.

Dietrich Hectors graduated as master in industrial sciences in Electromechanics at Group $\mathrm{T}$ international university college Leuven (Belgium) in 2001, and as master of science in Mechanical Engineering at the university of Leuven in 2003. Since 2004 he works towards a PhD in Electrical Engineering at the university of Leuven. In this $\mathrm{PhD}$ he was involved in several long term projects with industry. In 2009 he joined ArcelorMittal $R \& D$ Gent, where he is active as $R \& D$ Engineer.

François Henrotte graduated in engineering (Mechanics-Physics) in 1991 and received the $\mathrm{PhD}$ in Applied Sciences in 2000, at the University of Liège, Belgium. Since 2004, he is with the Institut of Electrical Machines in Aachen, Germany. His main research interests are the numerical modelling of electromechanical systems and magnetic materials, as well as the theoretical analysis of electromagnetic forces, differential geometry and applied mathematics. $\mathrm{He}$ is also active in projects with industry, for development and optimisation of electrical motors. He is the author of about 80 scientific publications.

Martin Hafner graduated in electrical engineering in 2006 at the RWTH Aachen University, Germany. His master thesis concerned the application of an energy-based vector hysteris model to timeharmonic finite element analysis. Since 2007 he is working as a research associate in the Institute of Electrical Machines

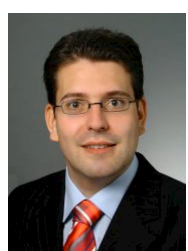
in Aachen, Germany. His research interests include the design and analysis of permanent magnet synchronous machines, hybrid FE-analytical electromagnetic machine models for rapid prototyping, and scientific visualization.

Mercedes Herranz Gracia received the M.Sc. degree in engineering (Mechanics-Electrotechnic) from the Technical University of Catalonia, Barcelona, Spain, in 2004. Since 2004, she has been a Researcher with the Institute of Electrical Machines, RWTH Aachen University and received her $\mathrm{PhD}$ on electrical engineering on 2008.

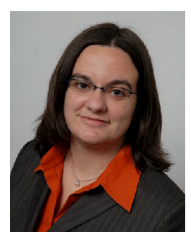
In this $\mathrm{PhD}$ she was involved in long-term projects with industry. Her main fields of research interest include numerical design, thermal aspects, and the acoustic behavior of electrical machines.

Kay Hameyer received his M.Sc. degree in electrical engineering from the University of Hannover and his Ph.D. degree from the Berlin University of Technology. He then worked with the Robert Bosch GmbH in Stuttgart as a Design Engineer for permanent magnet servo motors and board net components. Until 2004, he was a full Professor for Numerical Field Computations and Electrical Machines with the KU Leuven, Belgium. Currently, he is the Director of the IEM and holder of the chair Electromagnetic Energy

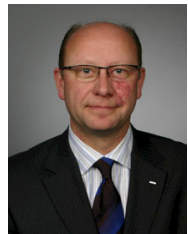
Conversion of the RWTH Aachen University, Germany. VDE, IEEE senior member. His research interests are numerical field computation, the design of electrical machines, and numerical optimisation strategies. 
Patrick Goes graduated at Ghent University as M.Sc. in physics in 1982 and as M.Sc. in philosophy in 1984. He joined the Ghent plant of the ArcelorMittal group where he was involved in numerical modelling and the development of EBT (Electron Beam texturing). In 2005 he joined ArcelorMittal Research Gent as R\&D enigineer numerical modelling. 